\title{
Cancer et environnement. L'humanité face à ses choix politiques
}

\section{Cancer and Environment. Humanity face to its political choices}

\author{
M.-F. Bacqué · J. Carretier
}

(C) Lavoisier SAS 2016

Qui se souvient de la campagne présidentielle de René Dumont en avril 1974 ? Le premier écologiste de la France d'alors aimait à se présenter avec un verre d'eau ou une pomme pour montrer la simplicité et la fragilité même de la Nature. Agronome, il était déjà sensible au problème de l'alimentation mondiale et prônait une baisse de la démographie.

Quarante ans plus tard, les menaces sur l'environnement s'aggravent et ce membre fondateur d'Attac ne serait pas surpris de constater les conséquences de la mondialisation en termes de pollution, de gaspillage et d'inégalités grandissantes entre les pays du Sud et du Nord. Il serait heureux d'apprendre en revanche que son nom est désormais attaché à la Coulée verte à Paris.

\section{L'environnement ? Un enjeu politique}

La Terre a continué de tourner et pourtant, malgré les débuts de l'écologie politique, le cycle de sa « consommation » virtuelle raccourcit d'année en année : ce 8 août 2016, nous avons absorbé toutes nos ressources naturelles et vivons " à crédit » sur un futur désormais bien précaire. Si les années 70 devinrent celles du début de la prise de conscience de l'anthropocène, l'action visible de l'homme sur la planète provoquait encore à l'époque un mouvement émotionnel. Aujourd'hui, les connaissances scientifiques peuvent être partagées par la majorité et deviennent un enjeu majeur, à la fois politique et économique. Étrangement, le facteur humain passe après ces considérations et les batailles que

M.-F. Bacqué $(\square)$

Rédactrice en chef de Psycho-Oncologie

EA 3071, université de Strasbourg, France

e-mail : mfbacque@club-internet.fr

J. Carretier $(\square)$

Département Cancer Environnement, centre Léon-Bérard,

28, rue Laennec, F-69008 Lyon, France

e-mail : julien.carretier@lyon.unicancer.fr se livrent les parlementaires, les lobbies, les gouvernements et les organisations non gouvernementales ne se déroulent pas sur le terrain direct de la démocratie (réclamé pourtant par de nombreuses voix sur internet), mais dans les alcôves et les sous-entendus. En effet, malgré les preuves, agir sur la pollution sous-entend en tout premier lieu, des compensations financières pour tous les abuseurs qui doivent modifier leurs systèmes de production. Et pire, en présence de liens ambigus mais bien réels entre l'émission d'un polluant et ses conséquences néfastes, les règlementations et les changements d'habitudes ne permettent même pas au principe de précaution de s'appliquer et entraînent encore pendant des années, maladie, handicap et mort.

\section{Informer est une question éthique}

Partager les connaissances faisait partie des rêves égalitaristes des premiers utopistes créateurs du réseau internet dans les années soixante. Nous sommes parvenus à développer cette extraordinaire possibilité, mais comment aller chercher, affronter puis synthétiser et intégrer toutes ces données ? Comment limiter les effets de la modification anthropique de notre environnement à l'origine d'une part conséquente de l'explosion des cancers ces trente dernières années ?

Les cliniciens, psychologues, psychiatres et oncologues observent, parfois impuissants, l'augmentation des questions des patients sur les liens entre le développement d'un cancer et les modifications environnementales. La pollution est devenue, pour la plupart, le nouvel agent insidieux qui attaque notre santé : à l'image des cellules cancéreuses, elle agit subrepticement à moyen terme et provoque l'impuissance du naïf qui la subit. Les médecins et les soignants, confrontés aux mêmes effets, sont aussi plongés dans les interrogations. Comment répondre, alors que la complexité oriente tous les résultats ? Comment éthiquement redonner de l'autonomie informative et, a minima, commenter avec les intéressés les connaissances issues de la recherche 
mondiale sur le sujet ? Accepter les enjeux politiques des effets de l'environnement sur la santé consiste bien, au niveau la relation médecin-malade, à écouter, à partager et à tirer des enseignements des études scientifiques qui, dans l'ici et maintenant, suggèrent certaines causalités.

Le site internet du centre Léon Bérard (cancer-environnement.fr) est totalement satisfaisant à cet égard : facile à consulter, avec des fiches parfaitement compréhensibles. Il répond à de nombreuses questions actuelles de la population, comme : « quels liens entre déodorants et cancer du sein ? Quels sont les effets du jeûne thérapeutique ? Quels sont les risques pour la santé de la consommation d'Aspartame?

Sur le plan économique pourtant, un tel portail d'informations sur les risques de cancer ne vient pas contrebalancer le pouvoir des décideurs. Cependant, il permet aux internautes de critiquer des assertions médiatiques ou personnelles et de retrouver leur capacité à penser leur destin.

\section{Les inégalités sociales sont là : les sans- diplômes font les plus sales boulots}

Qui n'a pas rencontré de patient en quête de causalité étiologique pour son cancer ou celui d'un proche ? C'est particulièrement flagrant dans le cas des cancers liés à une profession. Si les médecins mènent systématiquement l'enquête, les patients omettent parfois des rapprochements par simple ignorance. Ce sont fréquemment les veuves de ces ouvriers métallurgistes, chauffagistes, peintres, qui constituent a posteriori le dossier de demande de reconnaissance de maladie professionnelle en rétablissant la demande de pension de réversion de leur mari. Mais si certaines maladies sont évidentes comme dorénavant celles liées à l'amiante, les travailleurs précaires voient la reconnaissance de la leur sombrer dans l'oubli...

La main d'œuvre constituée par les migrants (appelés, dans les années 60 , les immigrés) apporte un immense bénéfice aux employeurs : ces personnes sans qualification recherchent à tout prix un travail (même mal rémunéré), elles ignorent les lois du pays, elles sont parfois illettrées, elles n'appartiennent pas à un réseau social, elles ne sauront donc pas faire appel aux juristes ou aux associations qui pourraient les aider en cas de préjudice.

Pourtant, les épidémiologistes estiment qu'il est parfois plus simple d'analyser les risques de cancers professionnels. Leur évaluation est beaucoup plus cadrée que dans un milieu domestique où les types de manipulation des produits cancérogènes sont vagues et leur chronologie peu précise. Les cancers professionnels sont souvent bien repérés par la médecine du travail ou les syndicats, ils concernent des groupes entiers de travailleurs.

La quatrième édition du Code européen contre le cancer est édifiante à ce niveau. Nous trouvons dans l'article de C. Espina et coll. la liste de tous les agents cancérogènes pour les professionnels qui manipulent mélanges, métaux, poussières et fibres dangereuses. Nous apprenons aussi que de nombreuses sources polluantes se trouvent dans les transports, le chauffage domestique, la cuisine, le nettoyage courant, les produits pour les bébés, etc.

\section{Mais comment distinguer les cancérogènes dans le cocktail nocif dans lequel tout habitant de la planète est dorénavant plongé ?}

Le CIRC $^{1}$ (ou IARC en anglais) s'est chargé de classer toutes les familles de produits cancérogènes lors d'expositions professionnelles ou domestiques. Cet ensemble de chercheurs a été confronté à de grandes difficultés d'évaluation : comment procéder quand les expositions aux cancérogènes sont mixtes? Quel poids attribuer aux autres pathologies tout aussi dangereuses ? Que faire de la pollution urbaine et de la pollution rurale ? Comment évaluer les populations transfrontalières qui subissent les nombreux passages polluants de camions de transport de marchandises ? Que traduire des émissions cancérogènes domestiques et des émissions professionnelles non liées à la manipulation de produits mais tout simplement au mode de chauffage ? Les inégalités sont encore reines entre les différents pays de l'Union Européenne (UE). Par exemple, tous les pays de l'Est, de la Bulgarie, à la Pologne, en passant par la Slovénie, la Slovaquie, la Hongrie, la République tchèque présentent des taux de matières particulaires (PM 10 et 2,5) dans l'air très élevés. Leurs législations retiennent peut-être ces risques mais leurs institutions sanitaires sont bien loin de pouvoir les prendre en compte... Enfin, les effets combinés des cancérogènes peuvent être additifs ou synergiques, et, ne l'oublions pas, tout une catégorie de travailleurs vulnérables, à temps partiel, sous-traitants, femmes et jeunes migrants n'est pas repérée ou ne bénéficie pas d'intervention parce qu'elle reste tout simplement invisible aux yeux du législateur et des médias. Nous sommes tous concernés par ces informations, mais même dans l'UE, nos règlementations et nos méthodes de protection sont disparates.

\section{Cancers et environnement : différents niveaux de réaction}

Après le scandale des logiciels fraudeurs du groupe Volkswagen qui ont trompé le monde entier sur la pollution des

\footnotetext{
${ }^{1}$ Le Centre international de recherche sur le cancer (CIRC), ou International Agency for Research on Cancer (IARC), est une agence intergouvernementale de recherche sur le cancer, créée en 1965 par l'Organisation mondiale de la santé (OMS) des Nations Unies. Ses bureaux sont situés à Lyon, en France. (www.iarc.fr)
} 
moteurs diesel de la marque et des marques du même constructeur (Audi, Porsche, Bentley, etc.), le doute est encore jeté sur les industriels. L'agro-industrie n'est pas en reste avec son refus de limiter les pesticides et les herbicides, ses antibiothérapies forcées des animaux à viande et ses mauvais traitements des poules pondeuses. Les vignerons ont aussi été attaqués pour leurs épandages massifs (ce sont les plus gros utilisateurs de produits phytosanitaires) près des écoles et des habitations. Les victimes ont pourtant tenté de porter en justice les effets de leur utilisation «naïve » de produits toxiques, mais les agriculteurs malades n'ont pas gagné leur procès. Comment comprendre en effet que la même industrie chimique puisse fabriquer les produits cancérogènes et nocifs et, en même temps, les médicaments pour les traiter? Un tel cynisme explique les combats sans cesse renouvelés des associations non gouvernementales. Hélas les États sont parties prenantes de tous ces enjeux. L'UE elle-même est la première à redonner du temps aux promoteurs des néonicotinoïdes, les « malheureux » doivent financer les modifications de leur appareil de production... Que préférez-vous, un travailleur malade, un retraité qui meurt précocement ou un chômeur (qui vote) en bonne santé ?

L'argument du chômage est devenu imparable pour les gouvernements qui " gèrent» au mieux les manifestations de routiers et chauffeurs de taxi, de producteurs de lait forcés au regroupement industriel et la liste est longue...

Psycho-Oncologie a choisi grâce à la coordination de Julien Carretier, docteur en Santé publique et responsable de l'information des publics au sein du département Cancer et Environnement du Centre Léon Bérard de Lyon, d'aborder ce sujet social, économique et politique, pour ses aspects psychologiques. Nous l'avons vu, les questions d'étiologie des cancers taraudent les patients, leurs proches et tous les citoyens. Ils peuvent dorénavant avoir accès à des sites informatifs et échanger avec leurs médecins et soignants sur ces sujets. Sur le plan psychologique encore, certains stéréotypes peuvent être déconstruits et les spécialistes en psychologie sociale n'auront que l'embarras du choix pour comprendre et limiter les phénomènes d'influence autour de ces questions taboues. Pour les psychothérapeutes d'orientation psychanalytique, la connaissance des effets objectifs de la pollution environnementale est aussi précieuse car elle occupe une place bien réelle dans les discours des malades. L'impact anxiogène de l'empoisonnement de l'air, de l'eau, de la nourriture est palpable dans tous les entretiens cliniques. Les accompagnements et les psychothérapies ne peuvent pas faire l'impasse de la réalité de la pollution de nos environnements professionnels et domestiques.

\section{Quels sont les niveaux d'action?}

Face à des systèmes de connaissance imbriqués (États, scientifiques indépendants, ONG critiques, décisions intergouvernementales), le citoyen semble pieds et poings liés. Pourtant, les systèmes d'accès à la connaissance sont là, il manque peut-être des médiateurs de ces connaissances, des systèmes de méta-connaissances qui ajusteraient au cas par cas les applications de ces résultats. Les différents articles sur les effets du surpoids dans le développement et le traitement de certains cancers le montre : l'information et l'accompagnement des patientes atteintes d'un cancer du sein est indispensable pour leur permettre suivi diététique et activité physique, mais ce sont également les liens sociaux retrouvés dans les groupes de malades qui facilitent l'adhésion au traitement et à la surveillance de la rémission.

Au niveau supérieur de l'information des sociétés, nous avons compris que seuls les groupements citoyens peuvent avoir voix au chapitre. Les class-actions à l'américaine doivent jouer le jeu en Europe. Le système des pétitions est efficace, celui de la représentation parlementaire doit être investi par un lobbying à l'échelle de celui des industriels. Le rôle des cliniciens consiste à témoigner des questions posées par les citoyens, le rôle des scientifiques à rechercher des niveaux de preuve, celui des spécialistes en sciences humaines à comprendre les effets individuels, de groupes et de société sur l'écologie mondiale, celui des politiques et, partant, de tous les citoyens, relève surtout de la capacité à s'informer de façon exhaustive et critique pour prendre les décisions partagées et responsables, celles qui mènent éthiquement à la meilleure vie pour tous au présent et surtout au futur... 